\title{
Erratum to: Potential of carbon nanotubes in algal biotechnology
}

\author{
May Dimova Lambreva ${ }^{1} \cdot$ Teresa Lavecchia $^{1,2} \cdot$ Esa Tyystjärvi $^{3}$. \\ Taras Kornelievich Antal $^{4} \cdot$ Silvia Orlanducci ${ }^{1,2} \cdot$ Andrea Margonelli ${ }^{1}$. \\ Giuseppina Rea ${ }^{1}$
}

Published online: 10 February 2016

(C) Springer Science+Business Media Dordrecht 2016

\section{Erratum to: Photosynth Res (2015) 125:451-471 \\ DOI 10.1007/s11120-015-0168-z}

The key to the graphs in Fig. 6b was incorrect in the original publication. The correct figure is shown below.

The online version of the original article can be found under doi:10.1007/s11120-015-0168-z.

\footnotetext{
May Dimova Lambreva maya.lambreva@mlib.ic.cnr.it

1 Institute of Crystallography, National Research Council of Italy, Via Salaria Km 29.300, 00015 Monterotondo Scalo, RM, Italy

2 Department of Chemical Science and Technology, University of Rome "Tor Vergata", Via della Ricerca Scientifica 1, 00133 Rome, Italy
}

3 Department of Biochemistry/Molecular Plant Biology, University of Turku, 20014 Turku, Finland

4 Department of Biophysics, Faculty of Biology, Lomonosov Moscow State University, Vorobyovi Gory bldg. 1/12, Moscow 119992, Russian Federation 


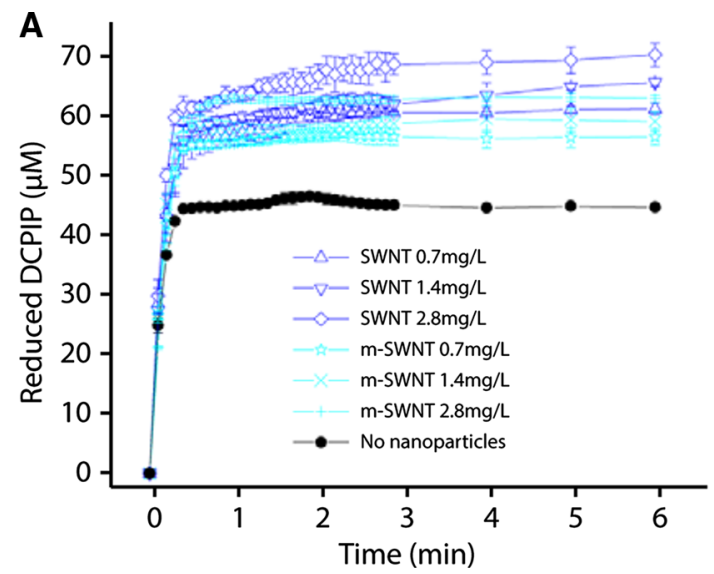

Fig. 6 Photosynthetic activity of chloroplasts supplemented with SWNTs and separate metallic SWNTs (m-SWNTs), measured as reduction of 2,6-dichlorophenol indophenol (DCPIP). a Chloroplasts with $\mathrm{m}$-SWNTs were less efficient in the reduction of DCPIP than assemblies with standard SWCNTs during the first $30 \mathrm{~min}$ of incubation. b In the long run, suspensions enriched with m-SWCNTs

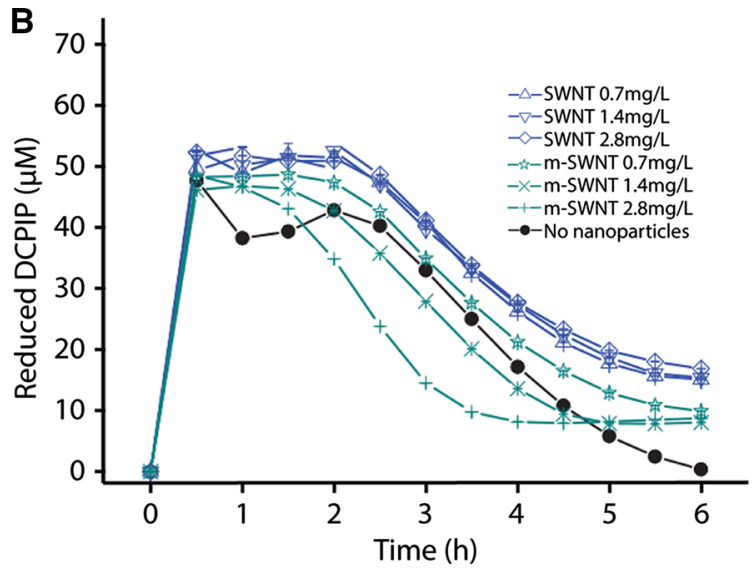

reduced less DCPIP than the control samples (without nanotubes), suggesting a competition for light energy between the photosynthetic pigments and nanotubes. Reprinted by permission from Macmillan Publishers Ltd: (Giraldo et al. 2014, doi:10.1038/nmat3890), copyright (2014) 